\title{
Multifunctional nanocomposite based on halloysite nanotubes for efficient luminescent bioimaging and magnetic resonance imaging
}

This article was published in the following Dove Press journal:

International Journal of Nanomedicine

19 September 2016

Number of times this article has been viewed

\author{
Tao Zhou' \\ Lei Jia' \\ Yi-Feng Luo ${ }^{2}$ \\ Jun $\mathrm{Xu}$ \\ Ru-Hua Chen ${ }^{2}$ \\ Zhi-Jun $\mathrm{Ge}^{2}$ \\ Tie-Liang $\mathrm{Ma}^{2}$ \\ Hong Chen ${ }^{2}$ \\ Tao-Feng Zhu ${ }^{2}$ \\ 'Department of Physics and \\ Chemistry, Henan Polytechnic \\ University, Jiaozuo, Henan, ${ }^{2}$ The \\ Affiliated Yixing Hospital of Jiangsu \\ University, Yixing, Jiangsu, People's \\ Republic of China
}

\begin{abstract}
A novel multifunctional halloysite nanotube (HNT)-based $\mathrm{Fe}_{3} \mathrm{O}_{4} @ \mathrm{HNT}$-polyethyleneimine-Tip-Eu(dibenzoylmethane) ${ }_{3}$ nanocomposite (Fe-HNT-Eu NC) with both photoluminescent and magnetic properties was fabricated by a simple one-step hydrothermal process combined with the coupling grafting method, which exhibited high suspension stability and excellent photophysical behavior. The as-prepared multifunctional Fe-HNT-Eu NC was characterized using various techniques. The results of cell viability assay, cell morphological observation, and in vivo toxicity assay indicated that the NC exhibited excellent biocompatibility over the studied concentration range, suggesting that the obtained Fe-HNT-Eu NC was a suitable material for bioimaging and biological applications in human hepatic adenocarcinoma cells. Furthermore, the biocompatible Fe-HNT-Eu NC displayed superparamagnetic behavior with high saturation magnetization and also functioned as a magnetic resonance imaging (MRI) contrast agent in vitro and in vivo. The results of the MRI tests indicated that the Fe-HNT-Eu NC can significantly decrease the $T_{2}$ signal intensity values of the normal liver tissue and thus make the boundary between the normal liver and transplanted cancer more distinct, thus effectively improving the diagnosis effect of cancers.
\end{abstract}

Keywords: halloysite nanotube, lanthanide complex, iron oxide, luminescence, contrast agent

\section{Introduction}

Multifunctional luminescent and magnetic nanomaterials have been widely used in biomedical applications such as magnetic resonance imaging (MRI), bioimaging, targeted drug delivery, magnetic hyperthermia, and cell labeling and separation. ${ }^{1-3}$ In recent years, organic dyes and quantum dots have been employed as biomedical fluorescent labels. However, these traditional fluorescent labels have limited use in large-scale applications due to their short lifetimes, photobleaching, and potential toxicity to cells. ${ }^{4,5}$ To address the limitations of organic dyes and quantum dots, lanthanide complexes have been investigated as luminescent probes because of their excellent optical properties, including sharp emission bands, high quantum yields, long lifetime, high color purity, and large Stokes shift. ${ }^{6,7}$ Unfortunately, the application of lanthanide complexes in biological imaging is limited by their low photochemical stability, hydrophobicity, and potential toxicity. ${ }^{8}$ To solve these issues, luminescent nanocomposites (NCs) have been designed by incorporating lanthanide complexes into matrices, thus generating suitable diagnostic bioimaging probes with the intrinsic stability of inorganic matrices and the luminescent features of trivalent lanthanide ions. $^{9-11}$ In recent years, nanoclays have attracted considerable interest as supports due to their low cost, small size, and unusual chemically active surfaces. ${ }^{12,13}$ 
Halloysite nanotube (HNT) is a two-layered aluminosilicate clay mineral with a chemical formula of $\mathrm{Al}_{2} \mathrm{Si}_{2} \mathrm{O}_{5}(\mathrm{OH})_{4} \cdot \mathrm{nH}_{2} \mathrm{O}$. It is a naturally occurring clay with a unique tubular nanostructure that has similar geometries to carbon nanotubes. Natural HNT clay has extremely high chemical stability, high surface area, and good adsorption properties. Because it is also environmentally friendly and biocompatible, it is suitable for biotechnological research. ${ }^{14-16}$ In contrast to other nanoscale synthetic supports, it is inexpensive and readily obtainable in the People's Republic of China. These factors make HNT clay an optimal candidate for a support material. We have recently fabricated a visible-light-induced claybased lanthanide polymer $\mathrm{NC}^{17}$ that has potential applications in cell imaging due to its excellent luminescence, high suspension stability, and low cytotoxicity.

MRI is an effective clinical tool used for the diagnosis of many diseases. It offers several advantages, including high accuracy, rapid scanning, and a long effective imaging window. It provides a wealth of physiological and anatomical information on healthy or diseased internal organs and tissues. ${ }^{18,19}$ Superparamagnetic iron oxide nanoparticles (NPs), such as $\mathrm{Fe}_{3} \mathrm{O}_{4}$ NPs, are strong enhancers of proton relaxation with superior magnetic resonance (MR) $T_{2}$ (transverse relaxation) shortening effects and can be used at a much lower concentration than paramagnetic agents. ${ }^{20,21}$ When synthesizing multifunctional magnetic and luminescent nanomaterials, we found that the luminescence intensity of luminescent labels dropped when coupled directly to $\mathrm{Fe}_{3} \mathrm{O}_{4}$ NPs. ${ }^{22}$ To avoid this quenching problem, we assembled $\mathrm{Fe}_{3} \mathrm{O}_{4} @$ HNTs and functionalized the $\mathrm{Fe}_{3} \mathrm{O}_{4} @$ halloysite with hydrophilic branched polyethyleneimine (PEI). We describe the applications of this multifunctional natural clay-based nanomaterial in bioimaging and MRI. To the best of our knowledge, this is the first example of magnetic natural clay-based lanthanide $\mathrm{Fe}_{3} \mathrm{O}_{4} @$ HNT-PEITip-Eu(dibenzoylmethane [DBM] $)_{3}$ NC (Fe-HNT-Eu NC) suitable for MRI and luminescent bioimaging; the fabrication route is shown in Figure 1. This low-cost preparation produced an NC material possessing high suspension stability, low cytotoxicity, efficient luminescent emission, long luminescence lifetime, high quantum yield, and improved photoluminescence stability. Meanwhile, the NC could function as an MRI contrast agent in vitro and in vivo. These attributes highlight the potential of the as-prepared $\mathrm{NC}$ for luminescent bioimaging and MRI.

\section{Materials and methods \\ Materials}

5-Bromothiophene-2-carbaldehyde and 1,10-phenanthroline5,6-dione were purchased from Acros Organics. 3-Iodopropyltrimethoxysilane (99\%) and halloysite were purchased from Sigma-Aldrich Co. (St Louis, MO, USA). DBM, analytic reagent, and branched $\mathrm{PEI}$ with a molecular mass of $25 \mathrm{kDa}$ were purchased from Aladdin Industrial Corporation. Europium chloride $\left(\mathrm{EuCl}_{3} \cdot 6 \mathrm{H}_{2} \mathrm{O}\right)$ was obtained by dissolving $\mathrm{Eu}_{2} \mathrm{O}_{3}$ (99.99\%; Shanghai Yuelong) in hydrochloric acid,

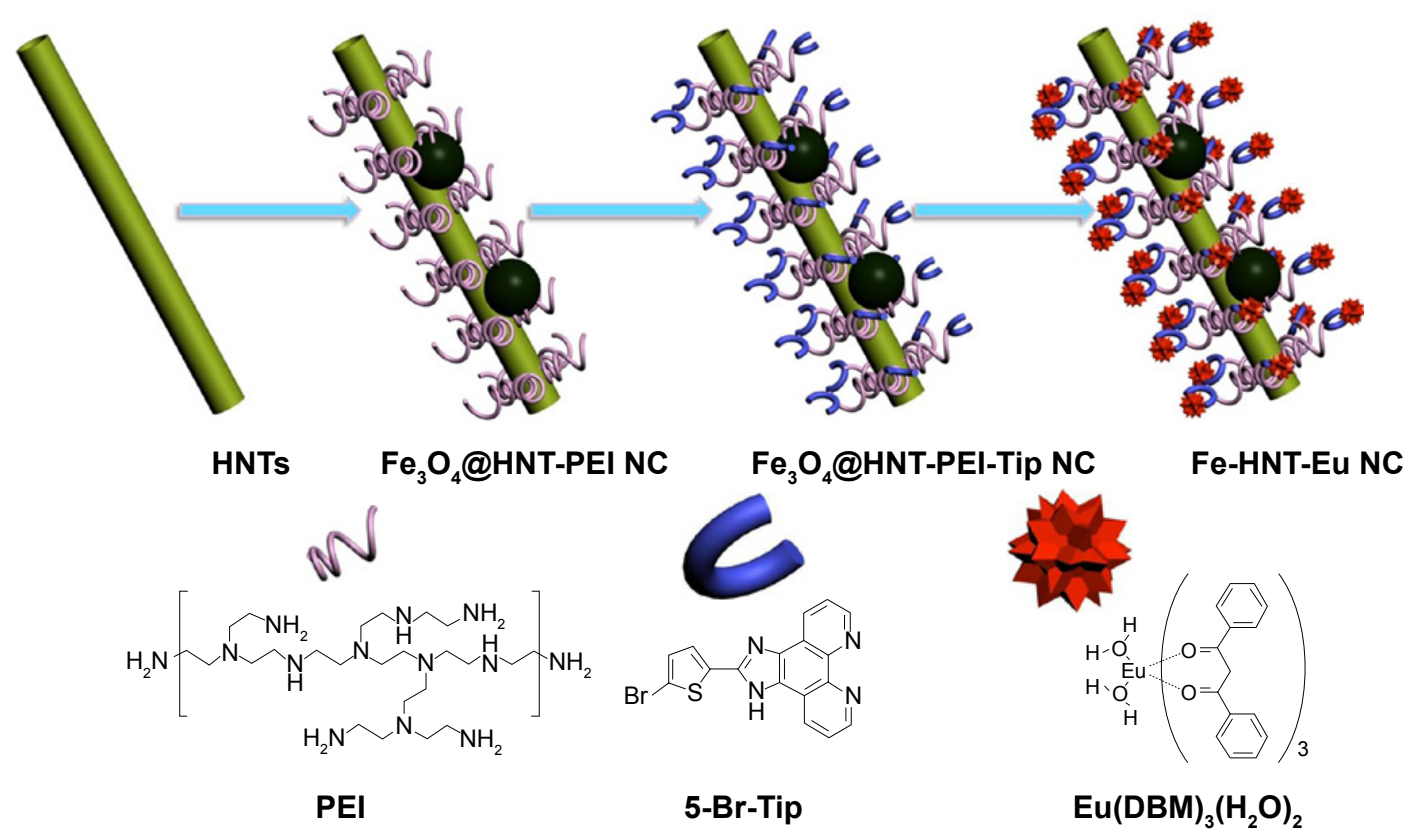

Figure I Schematic illustration of the synthesis of Fe-HNT-Eu NC.

Abbreviations: 5-Br-Tip, 2-(5-bromothiophen-2-yl)imidazo[4,5-f] [1,10]phenanthroline; DBM, dibenzoylmethane; HNT, halloysite nanotube; NC, nanocomposite; PEl, polyethyleneimine. 
followed by successive fuming to remove excess acid. All other reagents were analytically pure and were purchased from the China National Medicines Group.

Dulbecco's Modified Eagle's Medium (DMEM) and fetal bovine serum were purchased from Thermo Fisher Scientific (Waltham, MA, USA). The fluorescein Annexin V-FITC and propidium iodide (PI) double staining kit was purchased from BD Biosciences (San Jose, CA, USA). Cell Counting Kit-8 (CCK8) assay kits were purchased from Kaiji BioTech (Nanjing, People's Republic of China). VX2 liver tumor-bearing rabbits were purchased from the Second People's Hospital of Lianyungang.

\section{Synthesis}

\section{Amino modification of superparamagnetic Fe-HNT-Eu NC}

A three-step modification procedure was used to fabricate superparamagnetic $\mathrm{Fe}_{3} \mathrm{O}_{4} @$ HNT-PEI NC. ${ }^{23}$ First, $1 \mathrm{~g}$ of $\mathrm{FeCl}_{3} \cdot 6 \mathrm{H}_{2} \mathrm{O}$ was dissolved in $30 \mathrm{~mL}$ of ethylene glycol to form a clear solution. Then, $2.7 \mathrm{~g}$ of sodium acetate and $0.75 \mathrm{~g}$ of polyethylene glycol were added with constant stirring for 30 minutes. HNTs (300 mg) were then ultrasonically dispersed in the resulting dispersion for 3 hours. The mixture was sealed in a Teflon-lined stainless steel autoclave $\left(50 \mathrm{~mL}\right.$ capacity) and maintained at $200^{\circ} \mathrm{C}$ for 8 hours The resulting functionalized NPs, denoted as $\mathrm{Fe}_{3} \mathrm{O}_{4} @$ HNT, were then cooled to ambient temperature. The black magnetite particles were washed sequentially with ethanol and deionized water and dried under vacuum at $60^{\circ} \mathrm{C}$ for 24 hours. In the second step, $\mathrm{Fe}_{3} \mathrm{O}_{4} @$ HNT NPs (300 mg) were dispersed in dry toluene $(50 \mathrm{~mL})$ using an ultrasonic bath. 3-Iodopropyltrimethoxysilane $(0.5 \mathrm{~mL})$ was added to the $\mathrm{Fe}_{3} \mathrm{O}_{4} @$ HNT suspension, and the reaction mixture was heated to $110^{\circ} \mathrm{C}$ with mechanical stirring. After 8 hours, the solids were recovered using an $\mathrm{NdFeB}$ magnet, washed with ethanol three times to remove residual toluene and silane, and dried under vacuum. These NPs were denoted as $\mathrm{Fe}_{3} \mathrm{O}_{4} @$ HNT-I. Finally, in the third step, $\mathrm{Fe}_{3} \mathrm{O}_{4} @$ HNT-I NPs (300 mg) were suspended in $N, N$-dimethylformamide (DMF, $50 \mathrm{~mL})$ with sonication. PEI $(1.0 \mathrm{~mL}$ of a $50 \%$ aqueous solution) was added to the reaction mixture and heated to $130^{\circ} \mathrm{C}$ with mechanical stirring for 12 hours. The NPs were recovered using an $\mathrm{NdFeB}$ magnet, washed with ethanol three times to remove residual PEI, and dried under vacuum. These NPs were denoted as $\mathrm{Fe}_{3} \mathrm{O}_{4} @$ HNT-PEI.

\section{Synthesis of $\mathrm{Fe}_{3} \mathrm{O}_{4} @ H N T-P E I-T i p ~ N C$}

The ligand 2-(5-bromothiophen-2-yl)imidazo[4,5-f] [1,10] phenanthroline (5-Br-Tip) was synthesized according to methods reported in the literature. ${ }^{24} \mathrm{~A}$ mixture of 5-bromothiophene-2-carbaldehyde $(0.230 \mathrm{~g}, 1.2 \mathrm{mmol}), \mathrm{AcONH}_{4}$ (1.540 g, $1 \mathrm{mmol}$ ), and 1,10-phenanthroline-5,6-dione $(0.21 \mathrm{~g}, 1 \mathrm{mmol})$ in $\mathrm{AcOH}(20 \mathrm{~mL})$ was stirred and heated at $85^{\circ} \mathrm{C}$ for 4 hours. The mixture was then cooled to room temperature, and the product was precipitated during neutralization with $5 \mathrm{M} \mathrm{NH}_{4} \mathrm{OH}$. The precipitate was filtered, washed with $\mathrm{H}_{2} \mathrm{O}$ and ethanol, recrystallized from $\mathrm{MeOH}$, and dried under vacuum to yield a pure, light yellow product. $\mathrm{Fe}_{3} \mathrm{O}_{4} @$ HNT-PEI (300 mg) and $\mathrm{K}_{2} \mathrm{CO}_{3}(138 \mathrm{mg}, 1.0 \mathrm{mmol})$ were suspended in $30 \mathrm{~mL}$ of DMF, sonicated for 30 minutes, and then added to $20 \mathrm{~mL}$ of DMF containing the obtained 5-Br-Tip (381 mg, $1.0 \mathrm{mmol}$ ). The mixture was refluxed for 24 hours and centrifuged. After washing the resulting solids with DMF and ethanol (95\%) and drying at $60^{\circ} \mathrm{C}$, the $\mathrm{NC}$ $\mathrm{Fe}_{3} \mathrm{O}_{4} @$ HNT-PEI-Tip was obtained.

\section{Synthesis of $\mathrm{Fe}_{3} \mathrm{O}_{4} @ \mathrm{HNTs}-\mathrm{PEI}-\mathrm{Tip}-\mathrm{Eu}(\mathrm{DBM})_{3}$} The complex $\mathrm{Eu}(\mathrm{DBM})_{3}\left(\mathrm{H}_{2} \mathrm{O}\right)_{2}(43.0 \mathrm{mg}, 0.05 \mathrm{mmol}$, prepared from $\mathrm{DBM}$ and $\mathrm{EuCl}_{3} \cdot 6 \mathrm{H}_{2} \mathrm{O}$ according to the literature ${ }^{25}$ ) was refluxed with $300 \mathrm{mg}$ of $\mathrm{Fe}_{3} \mathrm{O}_{4} @$ HNT-PEITip in ethanol. The suspension was collected by centrifugation after 24 hours, and the excess unbounded complexes were washed away thoroughly with ethanol until no red emission was detected in the washing solution under ultraviolet (UV) excitation (365 nm). Fe-HNT-Eu NC was obtained after drying at $100^{\circ} \mathrm{C}$ for 3 hours.

\section{In vitro cell cytotoxicity, apoptosis, and imaging}

A CCK8 assay was used to evaluate the effect of the Fe-HNT-Eu NC on the survival of LO2 and HepG2 cells, a human hepatic cell line and human hepatic adenocarcinoma cell line, respectively. LO2 and HepG2 cells were obtained from the Cell Culture Center of the Basic Institute of Medical Sciences, Peking Union Medical College (Beijing, People's Republic of China). Cells were seeded into 96-well plates in DMEM at $1 \times 10^{4}$ cells/well. After overnight growth, cells were cultured with the Fe-HNT-Eu NC at concentrations of $400 \mu \mathrm{g} / \mathrm{mL}, 200 \mu \mathrm{g} / \mathrm{mL}, 100 \mu \mathrm{g} / \mathrm{mL}, 50 \mu \mathrm{g} / \mathrm{mL}, 25 \mu \mathrm{g} / \mathrm{mL}$, or $12.5 \mu \mathrm{g} / \mathrm{mL}$ for 24 hours. After 24 hours of incubation, $10 \mu \mathrm{L}$ CCK8 was added into each well and cells were incubated at $37^{\circ} \mathrm{C}$ for 4 hours. Finally, the optical density was measured at $450 \mathrm{~nm}$ using an F50 microplate reader. At least three parallel experiments were performed.

Apoptotic rates were determined by flow cytometry using an Annexin V/PI apoptosis kit. Briefly, LO2 cells were seeded overnight at a density of $1 \times 10^{6}$ cells/well in six-well plates and then treated for 24 hours with Fe-HNT-Eu NC 
at concentrations of $400 \mu \mathrm{g} / \mathrm{mL}, 200 \mu \mathrm{g} / \mathrm{mL}, 100 \mu \mathrm{g} / \mathrm{mL}$, or $50 \mu \mathrm{g} / \mathrm{mL}$. A total of $1 \times 10^{6}$ cells were collected by centrifugation and washed twice with cold phosphate-buffered saline. Annexin V-FITC/PI staining was performed according to the manufacturer's instructions, and the cells were analyzed using a BD Accuri C6 flow cytometer. At least three independent experiments were performed.

HepG2 cells were seeded in flat-bottomed six-well plates (Costar) in $2 \mathrm{~mL}$ of culture medium and incubated at $37^{\circ} \mathrm{C}$ with $5 \% \mathrm{CO}_{2}$. After overnight incubation, the cells were treated for 6 hours with the Fe-HNT-Eu NC at final concentrations of $50 \mu \mathrm{g} / \mathrm{mL}$, and then washed three times with phosphate-buffered saline. Cell membranes were observed by fluorescence imaging using a laser scanning upconversion luminescence microscope under excitation wavelengths of 340-380 nm.

\section{In vitro and in vivo MRI}

The experimental protocol was approved by the Jiangsu University Institutional Animal Care and Use Committee and performed in accordance with the International Guiding Prinfor Biomedical Research involving Animals-1985. To evaluate the effect of Fe-HNT-Eu NC on MR enhancement, dynamic contrast-enhanced MRI was performed in vivo in tumor-bearing rabbits using the same MRI equipment as the in vitro study. Rabbits were anesthetized using isoflurane (3\% loading dose, 1\% maintenance dose), and Fe-HNT-Eu $\mathrm{NC}(100 \mathrm{mg} / \mathrm{mL})$ was administered through the auricular vein at a dose of $16 \mathrm{mg} / \mathrm{kg}$ body weight. The rabbits were scanned dynamically before the experiment and also at different time points after injection (30 seconds, 3 minutes, 24 hours). The $T_{2}$-weighted sequence was as follows: repetition time $(\mathrm{TR})=2,549 \mathrm{~ms}$, echo time $(\mathrm{TE})=100 \mathrm{~ms}$, matrix $=224 \times 191$, field of view $(\mathrm{FOV})=180 \times 180$, thickness $=2.0 \mathrm{~mm}$, slice $=12$. For quantitative evaluation of the enhancement effect of the Fe-HNT-Eu NC, we obtained the $T_{2}$ signal intensity $\left(T_{2}\right.$-SI) of the normal liver, muscle and liver cancer tissues using the "region of interest" function in the Philips workstation. On the traverse $T_{2}$ images, regions of interest were manually delineated on the liver and muscle tissues, and the values of the SIs were then obtained automatically.

\section{In vivo toxicity}

After the rabbits were sacrificed, their organs (heart, liver, spleen, lung, and kidney) were excised, fixed in $4 \%$ formalin, paraffin embedded, and sectioned for hematoxylin and eosin staining for histopathological analysis. The slices were observed by an Eclipse E800 microscope.

\section{Methods of analysis and testing}

Powder X-ray diffraction patterns were determined by a Rigaku-Dmax 2400 diffractometer using $\mathrm{Cu} \mathrm{K} \alpha$ radiation over the $2 \theta$ range of $5^{\circ}-70^{\circ}$. Transmission electron microscopy (TEM) and energy-dispersive spectrometer (EDS) were performed using a Tecnai-G2-F30 microscope at an acceleration voltage of $300 \mathrm{kV}$. Magnetization measurements were performed on a vibrating sample magnetometer (LAKESHORE-7304; VSM, Model7304, Lake shore Cryotronics Inc., Westerville, OH, USA) at room temperature. Steady-state luminescence spectra and lifetime measurements were measured on an FSL920 fluorescence spectrometer (Edinburgh Instruments Ltd) with a $450 \mathrm{~W}$ Xe arc lamp as the steady-state excitation source and an Nd-pumped OPOlette laser as the excitation source for lifetime measurements. For photoluminescence stability measurements, monochromatic light (360 nm) was separated from the $450 \mathrm{~W}$ Xe arc lamp and was used as the irradiation source with a slit of $0.2 \mathrm{~nm}$ and a shutter opening after 10 seconds for 800 seconds. Solutions of Fe-HNT-Eu NC at different concentrations were evaluated by $T_{2}$-weighted MRI at 3.0 T using an eight-channel phased-array coil (Achieva 3.0T TX; Philips, Amsterdam, the Netherlands). The parameters of the $T_{2}$-weighted sequence were as follows: $\mathrm{TR}=2,549 \mathrm{~ms}$, TE $=100 \mathrm{~ms}$, matrix $=224 \times 191$, FOV $=120 \times 120$, thickness $=2.0 \mathrm{~mm}$, slice $=8$.

\section{Results and discussion Characterization of composition and microstructure}

We characterized the crystal structures of unmodified HNTs, as-synthesized $\mathrm{Fe}_{3} \mathrm{O}_{4} @ \mathrm{HNT}$ NC, and Fe-HNT-Eu $\mathrm{NC}$ using powder X-ray diffraction (Figure 2). For the

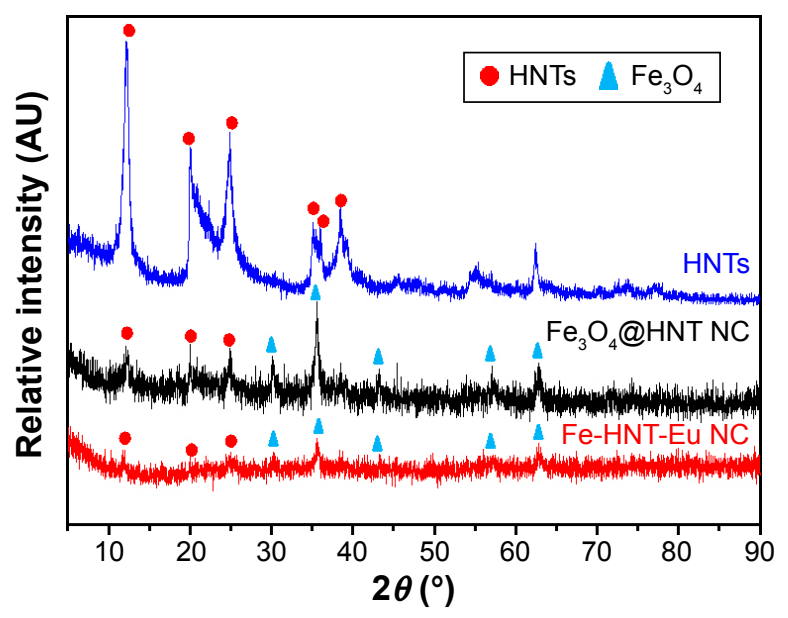

Figure 2 PXRD patterns of HNTs, $\mathrm{Fe}_{3} \mathrm{O}_{4} @ \mathrm{HNT} \mathrm{NC}$, and Fe-HNT-Eu NC. Abbreviations: HNT, halloysite nanotube; NC, nanocomposite; PXRD, powder X-ray diffraction. 
$\mathrm{Fe}_{3} \mathrm{O}_{4} @$ HNT NC, the presence of the characteristic diffraction peaks of HNTs indicates that the structure of the HNTs was maintained and that the synthetic reaction of $\mathrm{Fe}_{3} \mathrm{O}_{4}$ occurred on the surface but not in the interlayer. In addition to the characteristic diffraction peaks of HNTs, the $\mathrm{Fe}_{3} \mathrm{O}_{4} @$ HNT NC exhibited additional peaks that originated from cubic $\mathrm{Fe}_{3} \mathrm{O}_{4}$, which can be assigned to the (220), (311), (400), (422), (511), and (440) planes, according to the Joint Committee on Powder Diffraction Standards (JCPDS 19-0629). After modification by PEI and the lanthanide complex, the crystal structure of the Fe-HNT-Eu NC comprised the phases of cubic $\mathrm{Fe}_{3} \mathrm{O}_{4}$ and HNTs. The diffraction peaks corresponding to the crystalline layer were greatly reduced relative to those of $\mathrm{Fe}_{3} \mathrm{O}_{4} @$ HNT NC, indicating that PEI and the complex were successfully grafted onto the surface of the $\mathrm{Fe}_{3} \mathrm{O}_{4} @$ HNT NC. ${ }^{26}$

TEM images of the HNTs and Fe-HNT-Eu NC (Figure 3A and B) confirmed that the HNTs were cylindrical-shaped tubes with an open-ended lumen along the nanotube. The TEM images also indicated that the HNTs had an external
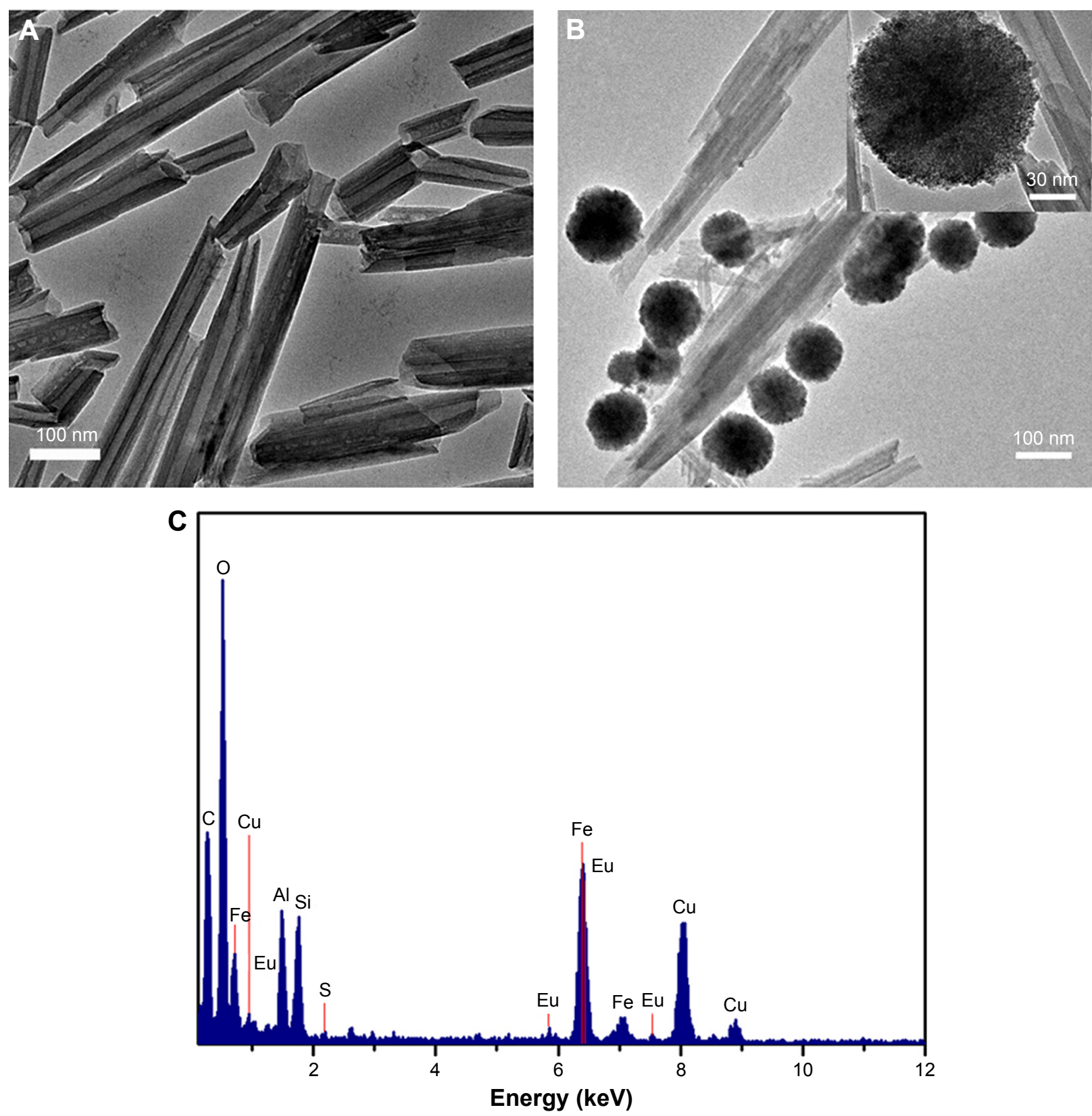

Figure 3 TEM image (A, B) and EDS spectrum (C) of Fe-HNT-Eu NC.

Note: The inset image is the high resolution of the Fe-HNT-Eu NC.

Abbreviations: EDS, energy-dispersive spectrometer; HNT, halloysite nanotube; NC, nanocomposite; TEM, transmission electron microscopy. 


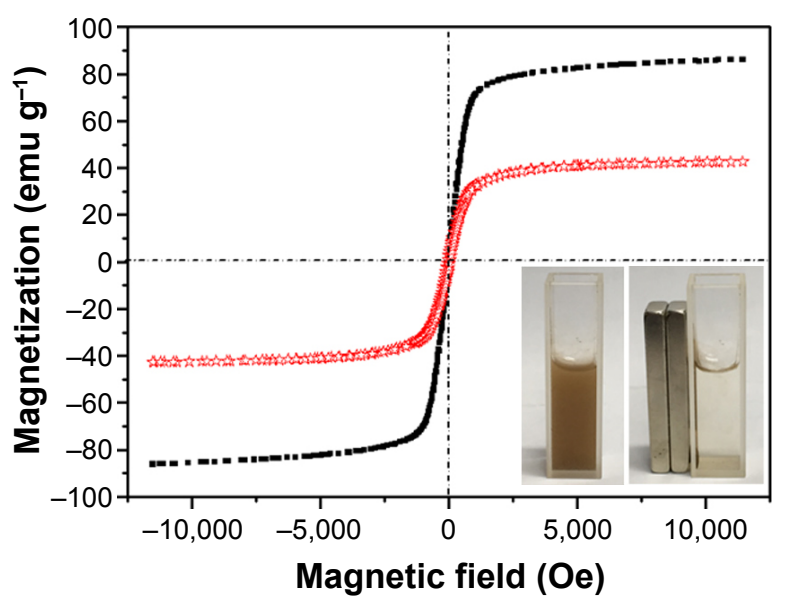

Figure 4 Magnetic hysteresis loops of pure $\mathrm{Fe}_{3} \mathrm{O}_{4} \mathrm{NP}(\boldsymbol{\square})$ and Fe-HNT-Eu NC (光). Note: The inset shows a photograph of Fe-HNT-Eu dispersed in water (left) and their magnetic response when placed in an external magnetic field (right).

Abbreviations: HNT, halloysite nanotube; NC, nanocomposite; NP, nanoparticle.

diameter of $\sim 60-200 \mathrm{~nm}$ and an inner diameter of $\sim 20-50 \mathrm{~nm}$. Black particles $\left(\mathrm{Fe}_{3} \mathrm{O}_{4} \mathrm{NPs}\right)$ with an average size of $100 \mathrm{~nm}$ were aggregated on the surface of the tube rather than in the lumen (Figure 3B). This assembly occurs because the HNTs can serve as nucleation precursors due to the high density of negative charges on their surface, which enables them to be modified by positive metal ions. In our system, iron(III) cations in solution attached to the surface of the HNTs and subsequently reduced into very fine magnetite particles in situ during hydrothermal treatment.

To confirm that $\mathrm{Fe}_{3} \mathrm{O}_{4} @$ HNTs were successfully modified by $\mathrm{Eu}$ (III) complexes, elemental mapping analysis of $\mathrm{Fe}_{3} \mathrm{O}_{4} @$ HNT and Fe-HNT-Eu NC was performed by EDS (Figure 3C).
Characteristic peaks ascribed to the elements $\mathrm{Al}, \mathrm{Si}, \mathrm{O}, \mathrm{Fe}, \mathrm{S}$, and Eu were observed in the EDS spectrum, indicating the successful formation of Fe-HNT-Eu NC.

The magnetic behaviors of the as-synthesized pure $\mathrm{Fe}_{3} \mathrm{O}_{4} \mathrm{NP}$ and Fe-HNT-Eu NC were studied using a vibrating sample magnetometer at room temperature. Field-dependent magnetization curves (Figure 4) showed negligible hysteresis loops, indicating that both nanomaterials exhibited characteristic superparamagnetic behavior at room temperature. The saturation magnetization of pure $\mathrm{Fe}_{3} \mathrm{O}_{4}$ NPs was $86.02 \mathrm{emu} \mathrm{g}^{-1}$, while that of Fe-HNT-Eu $\mathrm{NC}$ was $42.71 \mathrm{emu} \mathrm{g}^{-1}$ (Figure 4). The low magnetization value observed for the modified NC was due to the presence of nonmagnetic elements, such as HNTs, PEI, and Eu(III) complexes, in the conjugates. These data further reveal that the modification process does not drastically change the magnetic properties of the $\mathrm{Fe}_{3} \mathrm{O}_{4}$ NPs. The Fe-HNT-Eu $\mathrm{NC}$ possessed enough magnetic force to meet the needs of magnetic separation (Figure 4, inset).

\section{Analysis of photophysical behaviors of the composites}

Solid-state excitation (left) and emission (right) spectra of the Fe-HNT-Eu NC at room temperature are shown in Figure 5A. The excitation spectrum of the nanohybrids exhibits a wide wavelength range, extending from the UV region to the visible light region, with a peak at $360 \mathrm{~nm}$.

In the emission spectrum, the characteristic emissions of $\mathrm{Eu}(\mathrm{III})$ arising from the transition ${ }^{5} \mathrm{D}_{0} \rightarrow{ }^{7} \mathrm{~F}_{\mathrm{J}}(J=0,1,2,3,4)$ were detected under excitation at $360 \mathrm{~nm}$. Red emission from the
A

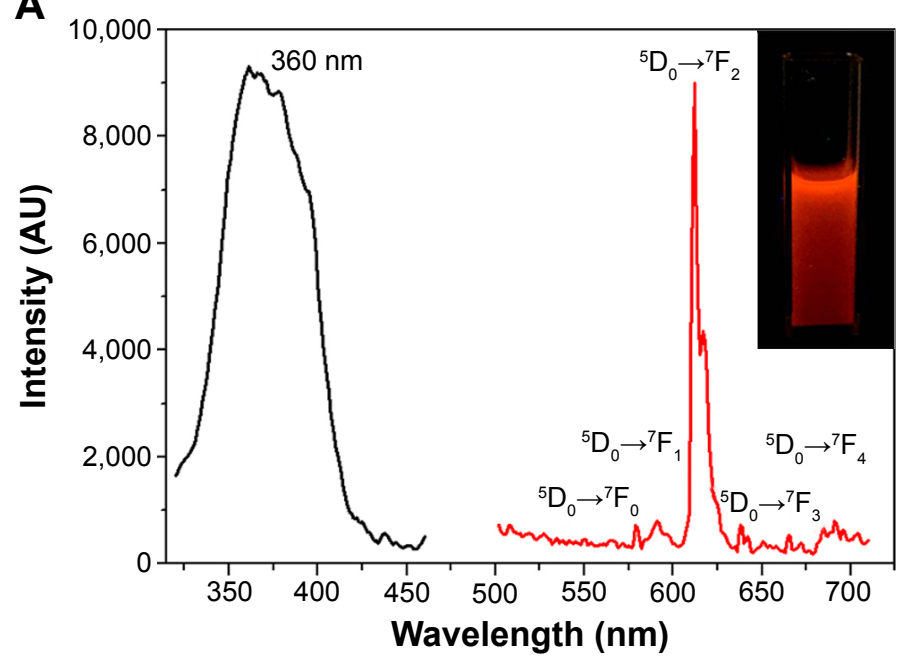

B

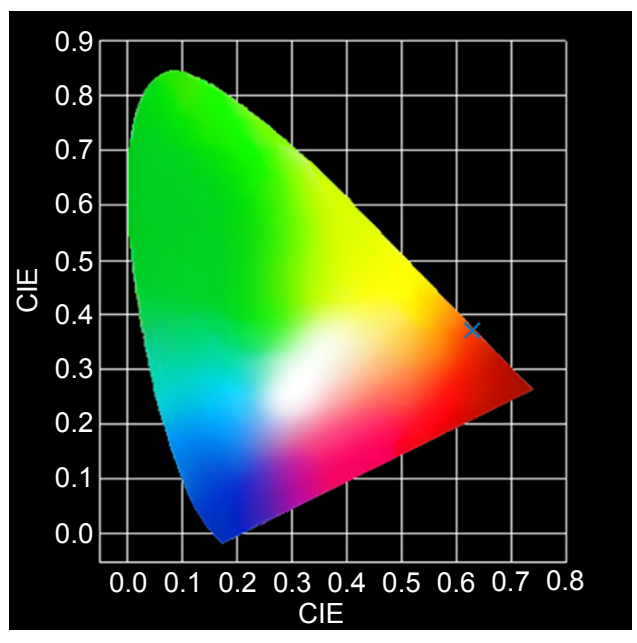

Figure 5 (A) Excitation and emission spectra of Fe-HNT-Eu NC, (B) CIE chromaticity diagram showing the $(x, y)$ photoluminescence emission color coordinates. The inset image shows the NC dissolved in water under UVA irradiation $(365 \mathrm{~nm})$.

Abbreviations: HNT, halloysite nanotube; NC, nanocomposite; UVA, ultraviolet A; CIE, Commission Internationale de l'Éclairage. 

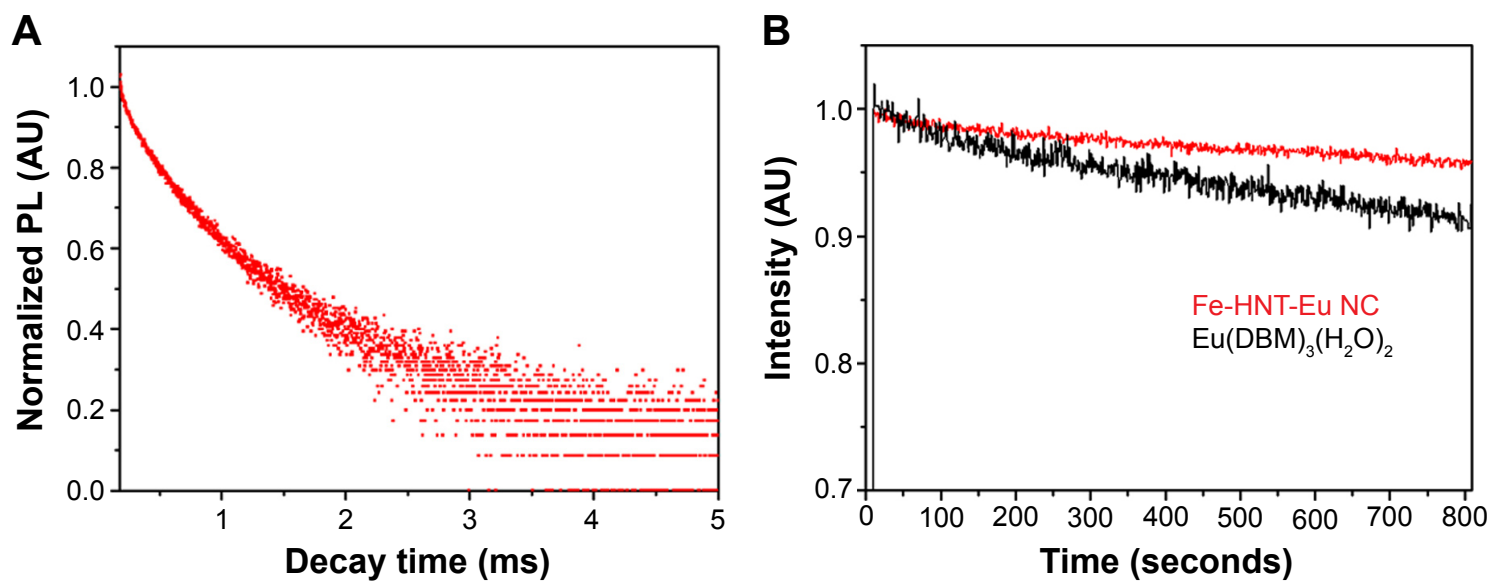

Figure 6 The emission decay curve and photoluminescence stability data.

Notes: $(\mathbf{A}){ }^{5} \mathrm{D}_{0}$ emission decay curve of Fe-HNT-Eu NC and (B) photoluminescence stability of Fe-HNT-Eu NC and the Eu(DBM) ${ }_{3}\left(\mathrm{H}_{2} \mathrm{O}\right)_{2}$ complex.

Abbreviations: DBM, dibenzoylmethane; HNT, halloysite nanotube; NC, nanocomposite; PL, photoluminescence.

dominant transition ${ }^{5} \mathrm{D}_{0} \rightarrow{ }^{7} \mathrm{~F}_{2}$ at $612 \mathrm{~nm}$ indicates that an efficient energy transfer from the ligands to the $\mathrm{Eu}(\mathrm{III})$ metal center occurred in the nanohybrids. The Commission Internationale de l'Éclairage (CIE) chromaticity coordinates for the Fe-HNT-Eu $\mathrm{NC}$ were computed on the basis of the emission spectrum and were found to be $(0.628,0.371)$ (Figure $5 \mathrm{~B})$. These values are very close to the standard CIE values of the National Television System Committee for red light $(x=0.670, y=0.330)$.

Luminescence lifetime is an important parameter for estimation of the luminescence efficiency of nanohybrids. The lifetime of the Fe-HNT-Eu NC was measured at room temperature by fitting to bi-exponential decay curves (Figure 6A). The fitting data (Table 1) show that two types of symmetrical Eu(III) sites exist in the nanohybrids. This can be attributed to site-to-site heterogeneity in the solid state. ${ }^{27}$ The ${ }^{5} \mathrm{D}_{0}$ lifetime of the $\mathrm{Eu}(\mathrm{DBM})_{3}\left(\mathrm{H}_{2} \mathrm{O}\right)_{2}$ complex was measured to be $53 \mu \mathrm{s} ;{ }^{28}$ the shortening of the lifetime may be due to the presence of absorbed $\mathrm{Eu}(\mathrm{DBM})_{3}\left(\mathrm{H}_{2} \mathrm{O}\right)_{2}$ on the surface of the matrix. Since multi-exponential decay curves are usually

Table I Photoluminescence data of composites containing Eu ${ }^{3+}$ in solids

\begin{tabular}{llll}
\hline Parameters & Fe-HNT-Eu NC & Parameters & Fe-HNT-Eu NC \\
\hline$\tau_{1}(\mathrm{~ms})^{\mathrm{a}}$ & $0.505(10.6 \%)$ & $I_{00}{ }^{\mathrm{c}}$ & 3,692 \\
$\tau_{2}(\mathrm{~ms})^{\mathrm{a}}$ & $0.335(89.4 \%)$ & $I_{01}{ }^{\mathrm{c}}$ & 10,370 \\
$<\tau>(\mathrm{ms})^{\mathrm{a}}$ & 0.310 & $I_{02}{ }^{\mathrm{c}}$ & 72,207 \\
$v_{00}\left(\mathrm{~cm}^{-1}\right)^{\mathrm{b}}$ & $17,27 \mathrm{I}$ & $\mathrm{I}_{03}{ }^{\mathrm{c}}$ & 4,905 \\
$v_{01}\left(\mathrm{~cm}^{-1}\right)^{\mathrm{b}}$ & 16,920 & $I_{04}{ }^{\mathrm{c}}$ & 11,583 \\
$v_{02}\left(\mathrm{~cm}^{-1}\right)^{\mathrm{b}}$ & 16,340 & $k_{\mathrm{r}}\left(\mathrm{ms}^{-1}\right)$ & 0.519 \\
$v_{03}\left(\mathrm{~cm}^{-1}\right)^{\mathrm{b}}$ & 15,674 & $k_{\mathrm{nr}}\left(\mathrm{ms}^{-1}\right)$ & $2.5 \mathrm{I}$ \\
$v_{04}\left(\mathrm{~cm}^{-1}\right)^{\mathrm{b}}$ & $14,45 \mathrm{I}$ & $q(\%)$ & 17.1
\end{tabular}

Notes: aThe lifetimes $(\tau)$ and average lifetime $\langle\tau\rangle$ of the ${ }^{5} \mathrm{D}_{0} \mathrm{Eu}^{3+}$ excited state obtained at room temperature. ${ }^{b}$ Energies of the ${ }^{5} D_{0} \rightarrow{ }^{7} F$, transitions $\left(v_{0}\right)$. Integrated

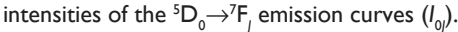

Abbreviations: HNT, halloysite nanotube; NC, nanocomposite. observed in these types of nanohybrids, ${ }^{29}$ the average lifetime $\langle\tau\rangle$ can be calculated using the following equation:

$$
<\tau>=\frac{\sum A_{i} \tau_{i}^{2}}{\sum A_{i} \tau_{i}}
$$

where $\tau_{i}$ is the component decay time and $A_{i}$ is the preexponential factor related to the statistical weight of each exponential. The Fe-HNT-Eu NC exhibited a longer average luminescence lifetime $(310 \mu \mathrm{s})$ than the corresponding $\mathrm{Eu}(\mathrm{DBM})_{3}\left(\mathrm{H}_{2} \mathrm{O}\right)_{2}$ complex, which indicates that the ternary europium NC was formed through the ligand exchange reaction. It also suggests that both the vibrations of the ligands around the Eu(III) metal center and the intermolecular collisions between the complexes were efficiently inhibited. The $\mathrm{NC}$ is expected to be suitable for use in cell imaging due to its long luminescence lifetime.

Quantum yield is another important parameter for estimation of the luminescence efficiency of the NC. We selectively determined the quantum yields $(q)$ of the $\mathrm{Eu}^{3+}$-containing $\mathrm{NC}$ by a previously described method (Table 1$) \cdot{ }^{30}$ Assuming that only nonradiative $\left(k_{\mathrm{nr}}\right)$ and radiative $\left(k_{\mathrm{r}}\right)$ processes are involved in the depopulation of the ${ }^{5} \mathrm{D}_{0}$ state, $q$ may be expressed as:

$$
q=\frac{k_{\mathrm{r}}}{k_{\mathrm{r}}+k_{\mathrm{nr}}}
$$

The relationship between the radiative processes, nonradiative processes, and experimental luminescence lifetime can be estimated through the following equation:

$$
k_{\mathrm{r}}+k_{\mathrm{nr}}=\frac{1}{\langle\tau\rangle}
$$


Therefore, quantum yields can be calculated from $k_{\mathrm{r}}$ and the experimental luminescence lifetime. The radiative transition rate $\left(k_{\mathrm{r}}\right)$ can be obtained by summing over the radiative rates $A_{0 J}$ for each of the ${ }^{5} \mathrm{D}_{0}-{ }^{7} \mathrm{~F}_{J}(J=0-4)$ transitions as follows:

$$
k_{\mathrm{r}}=\sum A_{0 J}=A_{00}+A_{01}+A_{02}+A_{03}+A_{04}
$$

The experimental coefficients of spontaneous emission $A_{0 J}$ can be calculated according to the following equation: ${ }^{31}$

$$
A_{0 J}=A_{01}\left(\frac{I_{0 J}}{I_{01}}\right)\left(\frac{v_{01}}{v_{0 J}}\right)
$$

where $A_{01}$ is the Einstein's coefficient of spontaneous emission between the ${ }^{5} \mathrm{D}_{0}$ and ${ }^{7} \mathrm{~F}_{1}$ energy levels. Under vacuum, the value of $A_{01(\mathrm{vac})}$ can be taken to be 14.65 second $^{-1}$. When using an average index of refraction of $\mathrm{n}=1.506$, the value of $A_{01}=\mathrm{n}^{3} A_{01(\mathrm{vac})} \approx 50$ second $^{-1},{ }^{32}$ where $v_{01}$ and $v_{0 J}\left(v_{0 J}=1 / \lambda_{J}\right)$ are the energy barycenters of the ${ }^{5} \mathrm{D}_{0}{ }_{7} \mathrm{~F}_{1}$ and ${ }_{5} \mathrm{D}_{0}{ }_{7} \mathrm{~F}_{\mathrm{J}}$ transitions $(\mathrm{J}=0-4)$, respectively. The emission intensity, $I$, taken as the integrated intensity $S$ of the ${ }^{5} \mathrm{D}_{0}-{ }^{7} \mathrm{~F}_{0-4}$ emission curves, can be defined as follows:

$$
I_{i-j}=\hbar \omega_{i-j} A_{i-j} N_{i} \approx S_{i-j}
$$

where $i$ and $j$ are the initial $\left({ }^{5} \mathrm{D}_{0}\right)$ and final levels $\left({ }^{7} \mathrm{~F}_{0-4}\right)$, respectively; $\hbar \omega_{i-j}$ is the transition energy; $A_{i-j}$ is the Einstein's coefficient of spontaneous emission; and $N_{i}$ is the population of the ${ }^{5} \mathrm{D}_{0}$-emitting level. ${ }^{33}$ The $\mathrm{NC}$ exhibited relatively high luminescence quantum yield (17.1\%), indicating its suitability for bio-labeling applications.

To evaluate the photoluminescence stability of the NC, we performed kinetic scans of luminescence induced by monochromic light $(360 \mathrm{~nm}$ ) partitioned from a $450 \mathrm{~W}$ Xe arc lamp. The dependence of the normalized emission intensity of the ${ }^{5} \mathrm{D}_{0} \rightarrow{ }^{7} \mathrm{~F}_{2}$ transition (612 nm) on irradiation time is shown in Figure 6B. The photoluminescence intensity of the Fe-HNT-Eu NC maintained $>95 \%$ of its initial value when monitored over a period of 800 seconds under high light intensity. Compared with complex $\mathrm{Eu}(\mathrm{DBM})_{3}\left(\mathrm{H}_{2} \mathrm{O}\right)_{2}$, the Fe-HNT-Eu NC showed no obvious luminescence decrease, indicating that the luminescent lanthanide ions are better protected against photobleaching when grafted onto the surface of a matrix.

\section{Cell cytotoxicity and apoptosis}

To guarantee the safety of Fe-HNT-Eu NC used as an imaging contrast agent, we assessed the effect of Fe-HNT-Eu NC on the viability of $\mathrm{LO} 2$ and $\mathrm{HepG} 2$ cells using a CCK8 assay.
Untreated cells were used as controls. We observed no significant negative effect on the viability of LO2 and HepG2 cells after 24 hours of incubation with various concentrations of Fe-HNT-Eu NC (Figure 7). Cell viability remained above $81 \%$ even at a concentration of $400 \mu \mathrm{g} / \mathrm{mL}$. These results indicated that Fe-HNT-Eu NCs have low cytotoxicity at the given concentration range, suggesting that they have favorable biocompatibility and the potential to be used as a medical agent. ${ }^{34}$

To further confirm that the NCs have no effect on the growth of human liver cells, Annexin V/PI staining and flow cytometry were used to quantify cell apoptosis. ${ }^{35}$ Treatment with NCs at a high concentration $(200 \mu \mathrm{g} / \mathrm{mL})$ had no effect on the number of apoptotic $\mathrm{LO} 2$ cells detected by flow cytometry, indicating that the prepared NCs were suitable for biological applications (Figure 8).

\section{In vitro cell imaging and MRI}

Earlier mentioned results suggest that Fe-HNT-Eu NC is suitable for bioimaging in living HepG2 cells. We observed HepG2 cells by fluorescence microscopy and bright field imaging under normal conditions (Figure 9A-C). HepG2 cells treated with $50 \mu \mathrm{g} / \mathrm{mL} \mathrm{Fe-HNT-Eu} \mathrm{NC} \mathrm{for} 6$ hours exhibited an intense red fluorescence signal (Figure 9B). The overlay image (Figure 9C) of the fluorescence (Figure 9B) and bright field (Figure 9C) images indicated that the $\mathrm{NC}$ signal was strongly correlated with the HepG2 cells. This suggests that the obtained Fe-HNT-Eu NC is a suitable material for bioimaging and biological applications. ${ }^{36}$ Earlier studies have reported that $\mathrm{Fe}_{3} \mathrm{O}_{4}$ can shorten $T_{2}$ relaxation time and that $\mathrm{Fe}_{3} \mathrm{O}_{4}$ NPs have great potential as MRI contrast agents. ${ }^{37-39}$ To investigate the MRI signal enhancement effects of the

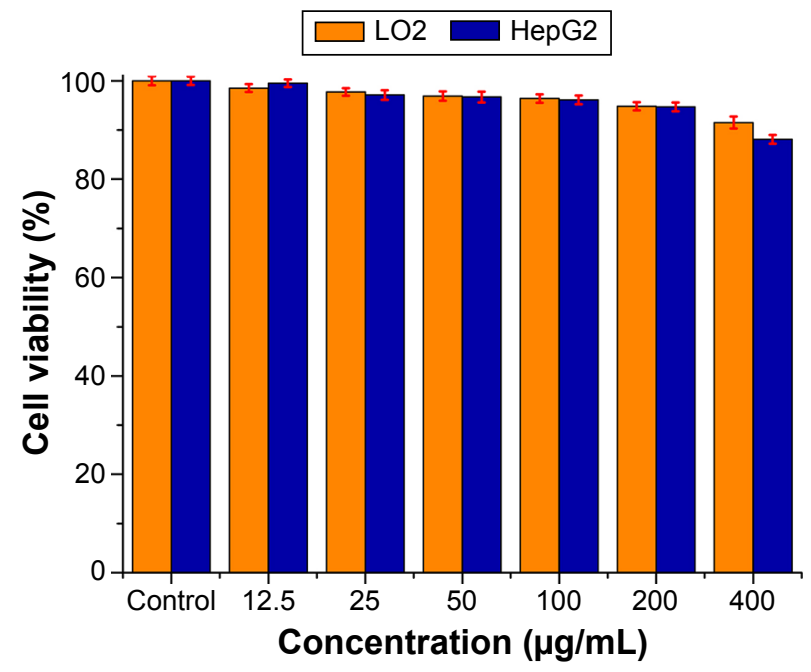

Figure 7 Viability of LO2 and HepG2 cells incubated with Fe-HNT-Eu NC at different concentrations for 24 hours.

Abbreviations: HNT, halloysite nanotube; NC, nanocomposite. 
A

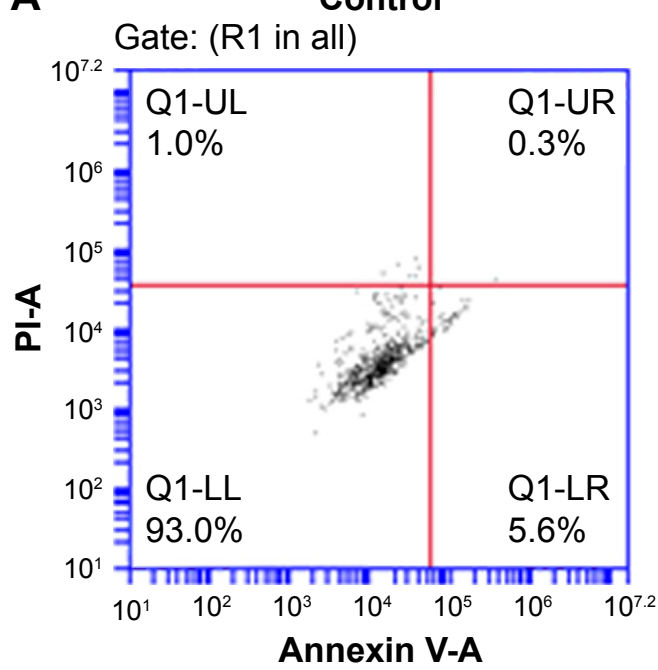

C

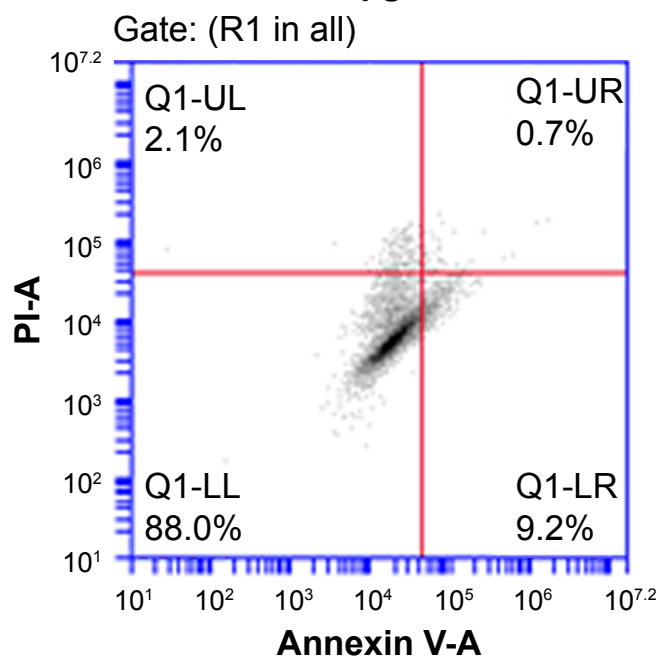

B

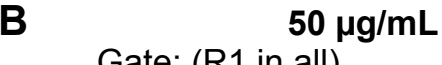

Gate: (R1 in all)

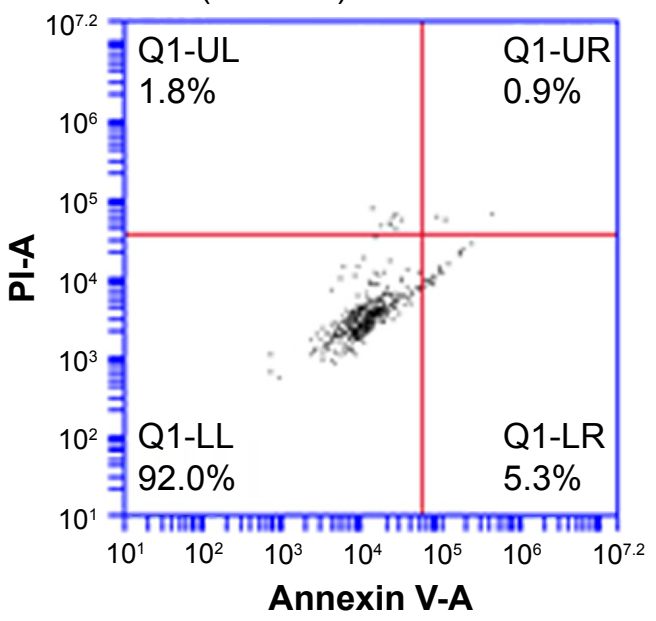

D

$200 \mu \mathrm{g} / \mathrm{mL}$

Gate: (R1 in all)

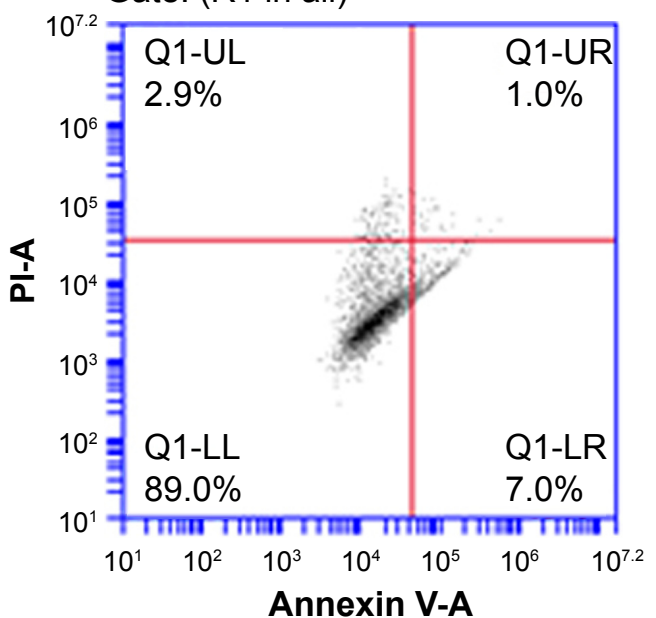

Figure 8 Annexin V/PI staining of LO2 cells incubated with Fe-HNT-Eu NC at $0 \mu g / \mathrm{mL}$ (A), $50 \mu g / \mathrm{mL}$ (B), $100 \mu g / \mathrm{mL}$ (C), and $200 \mu g / \mathrm{mL}$ (D) for 24 hours. Abbreviations: $\mathrm{HNT}$, halloysite nanotube; NC, nanocomposite; $\mathrm{PI}$, propidium iodide.
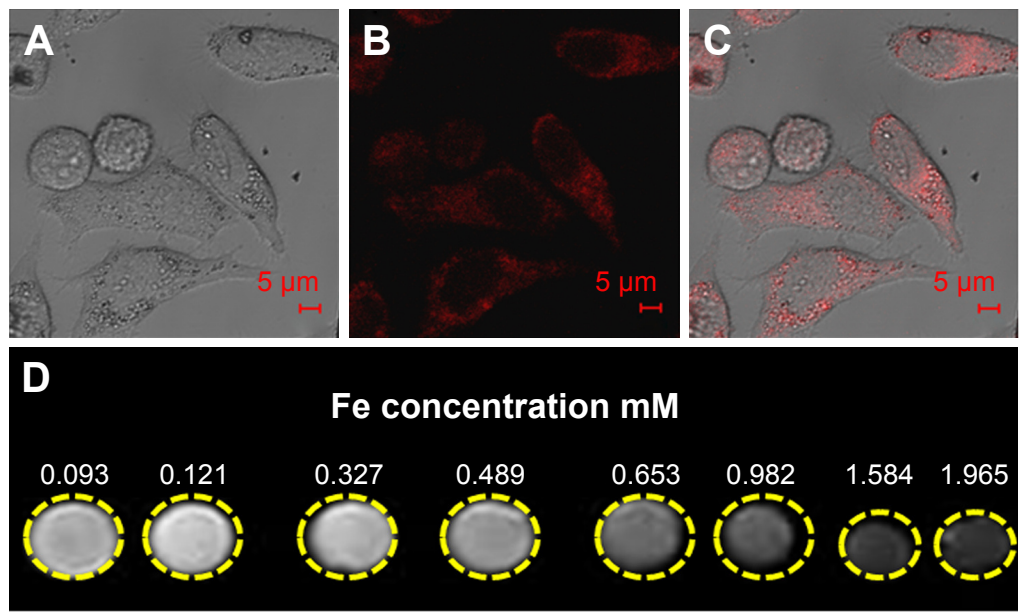

Figure 9 (A) Bright field image and fluorescence image; (B) HepG2 cells cocultured with Fe-HNT-Eu NC; (C) the overlapped image of images (A) and (B); (D) $T_{2}$-weighted MRI of Fe-HNT-Eu NC (the color change from light to dark indicates a gradual decrease in $T_{2}$-weighted MRI SI).

Abbreviations: HNT, halloysite nanotube; MRI, magnetic resonance imaging; NC, nanocomposite; SI, signal intensity. 
NCs prepared in this study, the transverse relaxation time $\left(T_{2}\right)$ of water protons in an aqueous solution of Fe-HNT-Eu NC was measured with a spin-echo pulse sequence. $T_{2}$-weighted images were obtained for an in vitro Fe-HNT-Eu NC solution and indicated that Fe-HNT-Eu NC can shorten the $T 2$ relaxation time and lower the $T_{2}$-SI. Test tubes containing Fe-HNT-Eu NC was represented with a gray/black color in the $T_{2}$-weighted images (Figure 9D). With increasing NC concentration, the $T_{2}$-SI gradually changed from light gray to dark gray. ${ }^{40}$ The gray value of the circles was reduced to $12.8 \%$ of its original levels by increasing the Fe concentrations from $0.093 \mathrm{mM}$ to $1.965 \mathrm{mM}$, which indicated that Fe-HNT-Eu NC generated MRI contrast in $T_{2}$-weighted sequences.

\section{In vivo $M R I$}

To confirm the $T_{2}$ imaging capability of Fe-HNT-Eu NC, we also investigated whether it can enhance the MRI signal of normal tissues in vivo. At first, Fe-HNT-Eu NC was injected at a dose of $0.185 \mathrm{mmol}$ [Fe] $\mathrm{kg}^{-1}$, and then the $T_{2}$-weighted images were recorded by alternate scanning in 24 hours. Dynamic contrast-enhanced MR was performed in normal rabbit liver and muscle tissue, and four time points were aquoted before (noncontrast phase) and after the enhancement (Figure 10A-D). From the enhanced magnitudes from Figure 10E, we can see that the $T_{2}$-SI values of the normal liver, which were in the range of 30-40, were significantly decreased compared with the SIs in the noncontrast phase (decreasing value is 43). While over 3 minutes, although the $T_{2}$-SI of the liver increased, the $T_{2}$-SI was still lower than the SI in the noncontrast phase (decreasing value is 12). In contrast, the $T_{2}$-SI values of the muscle did not change significantly at any time point. This difference in signal enhancement between the normal liver and muscle tissue was due to the significantly increased blood supply of the liver, which would increase the accumulation of the intravenously injected Fe-HNT-Eu NC in hepatic region. Over the 3-minute acquisition time of the $T_{2}$-weighted image, the concentration of Fe-HNT-Eu NC in the blood vessels of the rabbit muscle was not sufficiently high to decrease the $T_{2}$-SI. Thus, we hypothesize that the Fe-HNT-Eu NC can not only enhance the $T_{2}$-weighted SI of normal liver but also indicate differences in biological characteristics (such as blood supply) between different tissues.

Furthermore, in transplanted liver cancer, the blood supply of cancerous liver is lower than that of normal liver. In addition, a fiber diolame forms around the cancerous tissue. However, the gray value of the red region in Figure 10B was still reduced to $56.6 \%$ of its original levels in Figure 10A. Earlier mentioned facts can help us to conclude that the boundary between the normal liver and transplanted cancer was more distinct in the $T_{2}$-weighted images after enhancement with Fe-HNT-Eu NC, suggesting that Fe-HNT-Eu NC can be used for tumor discovery and diagnosis.

In addition, 24 hours after the injection of the Fe-HNT-Eu NC, the $T_{2}$-SI of the normal liver was recovered to similar levels as in the noncontrast phase (Figure 10D), suggesting that the Fe-HNT-Eu NC can be metabolized effectively without leaving any residual material in the organism.
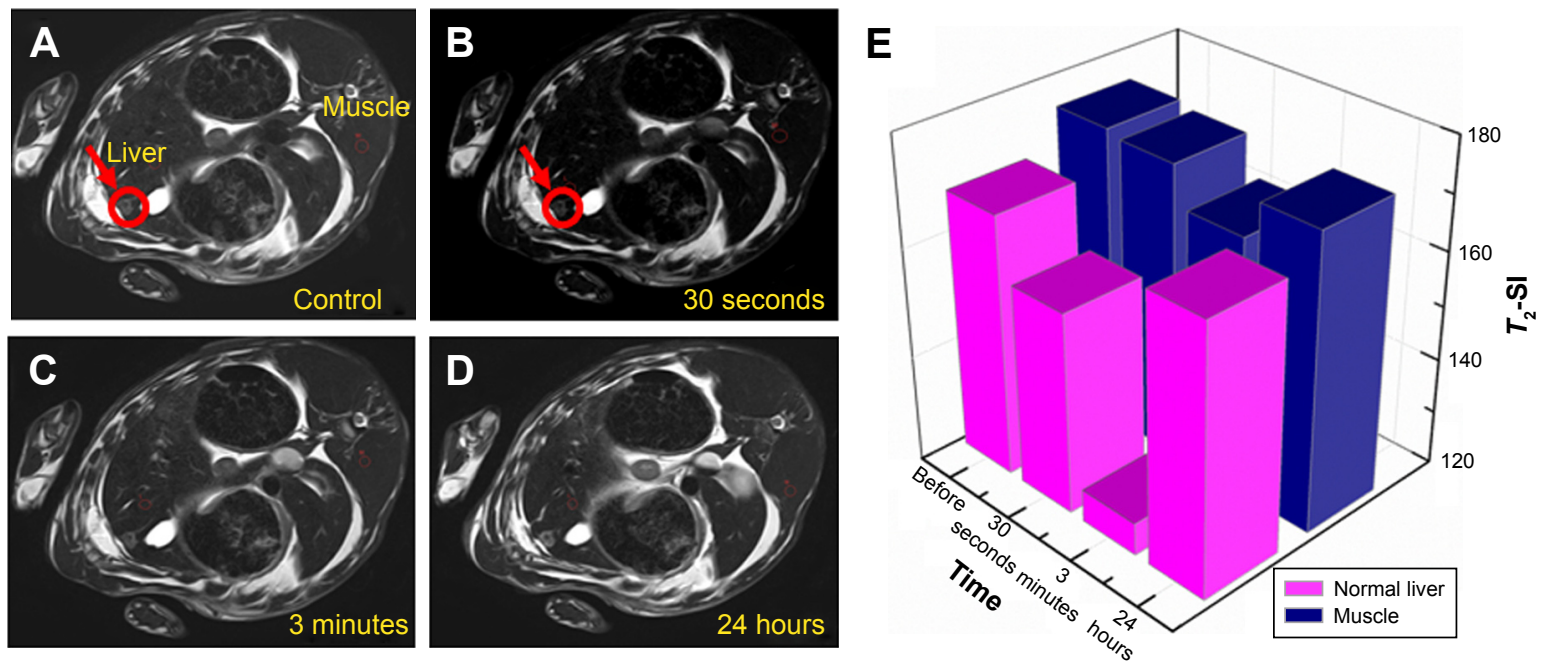

Figure 10 (A-D) $T_{2}$-weighted MR images of normal liver, muscle, and tissues before and after the injection of Fe-HNT-Eu NC for 30 seconds, 3 minutes, and 24 hours in rabbit. The red marker highlights the change in SI of the tumor before and after the MRI. (E) Mean intensity of $T_{2}$-weighted MR signals of normal liver and muscle at different times.

Abbreviations: HNT, halloysite nanotube; MR, magnetic resonance; MRI, MR imaging; NC, nanocomposite; SI, signal intensity. 


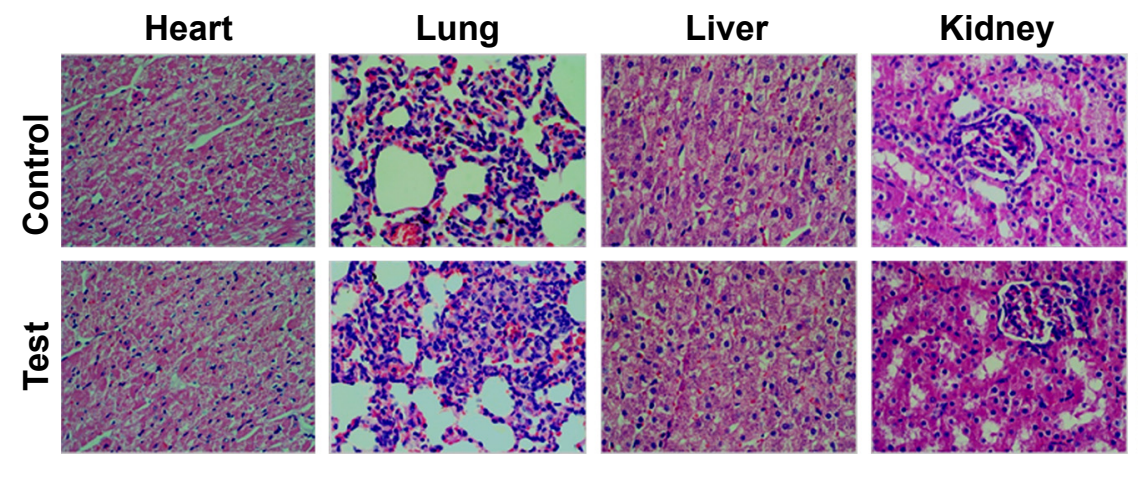

Figure I I Histopathological analysis of rabbit heart, lung, liver, kidney, and spleen tissues after the injection with Fe-HNT-Eu NC. Abbreviations: HNT, halloysite nanotube; NC, nanocomposite.

Overall, the current study demonstrated that Fe-HNT-Eu NCs can serve as in vitro and in vivo MRI contrast enhancers, can help distinguish between different normal tissues, and can help distinguish tumor tissues from normal tissues. These results suggest that Fe-HNT-Eu NCs have great potential as contrast agents in $T_{2}$-weighted MRI.

\section{In vivo toxicity}

We performed histopathological analysis on various tissues of rabbits to determine whether the NC caused any acute toxicity after 1-week injection of Fe-HNT-Eu NC. Analysis of tissue damage, inflammation, and lesions was performed on hematoxylin-and-eosin-stained sections of the heart, liver, spleen, lung, and kidney. Neither noticeable organ damage nor inflammation was observed in treated tissues compared with a control group (Figure 11). These results indicated that there was no significant toxicity of Fe-HNT-Eu NC at the exposure times used in this study, further demonstrating that Fe-HNT-Eu NCs have low toxicity and are safe for use in vivo.

\section{Conclusion}

A dual-modal nanoprobe Fe-HNT-Eu NC with luminescent bioimaging and $T_{2}$-weighted MRI was prepared and investigated in detail. The prepared HNT-based magnetic luminescent $\mathrm{NC}$ exhibited high suspension stability, low cytotoxicity, and excellent photophysical behaviors, which provided opportunities for luminescent bioimaging. The evaluation of Fe-HNT-Eu NC for contrast agent was conducted by in vitro cytotoxicity and apoptosis assay, in vitro cell imaging, in vitro and in vivo MRI, and in vivo toxicity studies in the rabbits. The results suggested that the biocompatible Fe-HNT-Eu NC prepared in this research can be used for tumor discovery and diagnosis.

\section{Acknowledgments}

This work was supported in part by the National Natural Science Foundation of China (No 21404033, No 21401046), the Technology Research Project of Henan Province (152102210314), the Foundation of the Social Development Project of Jiangsu (BE2015621), and the Natural Science Foundation of Jiangsu (BK20141122).

\section{Disclosure}

The authors report no conflicts of interest in this work.

\section{References}

1. Yan K, Li H, Wang X, et al. Self-assembled magnetic luminescent hybrid micelles containing rare earth Eu for dual-modality MR and optical imaging. J Mater Chem B. 2014;2(5):546-555.

2. Zhu H, Shang Y, Wang W, et al. Fluorescent magnetic $\mathrm{Fe}_{3} \mathrm{O}_{4} /$ rare earth colloidal nanoparticles for dual-modality imaging. Small. 2013; 9(17):2991-3000.

3. Xu H, Cheng L, Wang C, Ma X, Li Y, Liu Z. Polymer encapsulated upconversion nanoparticle/iron oxide nanocomposites for multimodal imaging and magnetic targeted drug delivery. Biomaterials. 2011; 32(35):9364-9373.

4. Ma Q, Nakane Y, Mori Y, et al. Multilayered, core/shell nanoprobes based on magnetic ferric oxide particles and quantum dots for multimodality imaging of breast cancer tumors. Biomaterials. 2012;33(33): 8486-8494.

5. Park JC, Yu MK, An GI, et al. Facile preparation of a hybrid nanoprobe for triple-modality optical/PET/MR imaging. Small. 2010;6(24): 2863-2868.

6. Ma Y, Wang Y. Recent advances in the sensitized luminescence of organic europium complexes. Coord Chem Rev. 2010;254(9-10):972-990.

7. Bünzli JCG, Svetlana VE. Basics of lanthanide photophysics. In: Hänninen P, Härmä H, editors. Lanthanide Luminescence: Photophysical, Analytical and Biological Aspects. Berlin: Springer; 2011:1-47.

8. Zhang Y, Wei W, Das GK, Thatt T, Tan Y. Engineering lanthanide-based materials for nanomedicine. J Photochem Photobiol C. 2014;20(9): 71-96.

9. Comby S, Surender EM, Kotova O, Truman LK, Molloy JK, Gunnlaugsson T. Lanthanide-functionalized nanoparticles as MRI and luminescent probes for sensing and/or imaging applications. Inorg Chem. 2014;53(4):1867-1879.

10. Dong H, Du SR, Zheng XY, et al. Lanthanide nanoparticles: from design toward bioimaging and therapy. Chem Rev. 2015;115(19): 10725-10815. 
11. Carlos LD, Ferreira RAS, de Zea Bermudez V, Julián-López B, Escribano P. Progress on lanthanide-based organic-inorganic hybrid phosphors. Chem Soc Rev. 2011;40(2):536-549.

12. Serefoglou E, Litina K. Smectite clays as solid supports for immobilization of $\beta$-glucosidase: synthesis, characterization, and biochemical properties. Chem Mater. 2008;20(12):4106-4115.

13. Tzialla AA, Pavlidis IV. Lipase immobilization on smectite nanoclays: characterization and application to the epoxidation of $\alpha$-pinene. Bioresour Technol. 2010;101(6):1587-1594.

14. Vergaro V, Abdullayev E, Cingolani R, Lvov Y, Leporatti S. Halloysite clay nanotubes: characterization and biocompatibility study. Biomacromolecules. 2010;11(9):820-828.

15. Islam MR, Bach LG, Lim KT. Poly(2-hydroxyethyl methacrylate) grafted halloysite nanotubes as a molecular host matrix for luminescent ions prepared by surface-initiated RAFT polymerization and coordination chemistry. Appl Surf Sci. 2013;276(7):298-305.

16. Cavallaro G, Donato D, Lazzara G, Milioto S. Films of halloysite nanotubes sandwiched between two layers of biopolymer: from the morphology to the dielectric, thermal, transparency, and wettability properties. J Phys Chem C. 2011;115(42):20491-20498.

17. Jia L, Zhou T, Xu J, et al. Visible light-induced lanthanide polymer nanocomposites based on clays for bioimaging applications. JMater Sci. 2016;51:1324-1332.

18. Zhou Z, Sun Y, Shen J, et al. Iron/iron oxide core/shell nanoparticles for magnetic targeting MRI and near-infrared photothermal therapy. Biomaterials. 2014;35(26):7470-7478.

19. Kim SM, Im GH, Lee DG, Lee JH, Lee WJ, Lee IS. $\mathrm{Mn}^{2+}$-doped silica nanoparticles for hepatocyte-targeted detection of liver cancer in T-1-weighted MRI. Biomaterials. 2013;34(35):8941-8948.

20. Yang G, Gong H, Liu T, Sun X, Cheng L, Liu Z. Two-dimensional magnetic WS $@ \mathrm{Fe}_{3} \mathrm{O}_{4}$ nanocomposite with mesoporous silica coating for drug delivery and imaging-guided therapy of cancer. Biomaterials. 2015;60:62-71.

21. He W, Cheng L, Zhang L, Liu Z, Cheng Z, Zhu X. Facile fabrication of biocompatible and tunable multifunctional nanomaterials via iron-mediated atom transfer radical polymerization with activators generated by electron transfer. ACS Appl Mater Interfaces. 2013;5(19): 9663-9669.

22. Li MJ, Chen Z, Yam VWW, Zu Y. Multifunctional ruthenium(II) polypyridine complex-based core-shell magnetic silica nanocomposites: magnetism, luminescence, and electrochemiluminescence. ACS Nano. 2008;2(5s):905-912.

23. Yiu HHP, Pickard MR, Olariu CI, Williams SR, Chari DM, Rosseinsky MJ. $\mathrm{Fe}_{3} \mathrm{O}_{4}$-PEI-RITC magnetic nanoparticles with imaging and gene transfer capability: development of a tool for neural cell transplantation therapies. Pharm Res. 2012;29(5):1328-1343.

24. Zheng CG, Ma CL, Yu XW. Electronic effect of substituents on the DNA intercalation of ruthenium(II) polypyridyl complexes. Chem Biodiversity. 2011;8(8):1486-1496.
25. Melby LR, Rose NJ, Abramson E, Caris JC. Synthesis and fluorescence of some trivalent lanthanide complexes. J Am Chem Soc. 1964;86(23): 5117-5125.

26. Zhao DF, Zhou J, Liu N. Surface characteristics and photoactivity of silver-modified palygorskite clays coated with nanosized titanium dioxide particles. Mater Charact. 2007;58(3):249-255.

27. Guo XM, Wang XM, Zhang HJ, et al. Preparation and luminescence properties of covalent linking of luminescent ternary europium complexes on periodic mesoporous organosilica. Microporous Mesoporous Mater. 2008;116(1-3):28-35.

28. Singh AK, Singh SK, Mishra H, Prakash R, Rai SB. Structural, thermal, and fluorescence properties of $\mathrm{Eu}(\mathrm{DBM})_{3} \mathrm{Phen}_{\chi}$ complex doped in PMMA. J Phys Chem B. 2010;114(41):13042-13051.

29. Wen X, Li M, Wang Y, et al. Colloidal nanoparticles of a europium complex with enhanced luminescent properties. Langmuir. 2008;24(13): 6932-6936.

30. Werts MHV, Jukes RTF, Verhoeven JW. The emission spectrum and the radiative lifetime of $\mathrm{Eu}^{3+}$ in luminescent lanthanide complexes. Phys Chem Chem Phys. 2002;4(9):1542-1548.

31. Teotonio EES, Espínola JGP, Brito HF, et al. Influence of the $\mathrm{N}$-[methylpyridyl]acetamide ligands on the photoluminescent properties of Eu(III)-perchlorate complexes. Polyhedron. 2002;21(18):1837-1844.

32. Hazenkamp MF, Blasse G. Rare-earth ions adsorbed onto porous glass: luminescence as a characterizing tool. Chem Mater. 1990;2(2): 105-110.

33. Malta OL, Couto dos Santos MA, Thompson LC, Ito NK. Intensity parameters of $4 \mathrm{f}-4 \mathrm{f}$ transitions in the $\mathrm{Eu}$ (dipivaloylmethanate) 1, 10-phenanthroline complex. J Lumin. 1996;69(2):77-84.

34. Xu J, Sun Z, Jia L, et al. Visible light sensitized attapulgite-based lanthanide composites: microstructure, photophysical behaviour and biological application. Dalton Trans. 2011;40(48):12909-12916.

35. Ma T, Xu J, Wang Y, et al. Ternary copper(II) complexes with amino acid chains and heterocyclic bases: DNA binding, cytotoxic and cell apoptosis induction properties. J Inorg Biochem. 2015;144:38-46.

36. Wang Y, Chang H, Jia L, et al. Development of a visible-light-sensitized THA-based lanthanide nanocomposite for cell imaging. Mater Lett. 2015; 161:644-647.

37. Yang $\mathrm{P}$, Wang $\mathrm{F}$, Luo $\mathrm{X}$, et al. Rational design of magnetic nanorattles as contrast agents for ultrasound/magnetic resonance dual-modality imaging. ACS Appl Mater Interfaces. 2014;6(15):12581-12587.

38. Yang J, Luo Y, Xu Y, et al. Conjugation of iron oxide nanoparticles with RGD-modified dendrimers for targeted tumor MR imaging. ACS Appl Mater Interfaces. 2015;7(9):5420-5428.

39. Wang G, Zhang X, Skallberg AS, et al. One-step synthesis of waterdispersible ultra-small $\mathrm{Fe}_{3} \mathrm{O}_{4}$ nanoparticles as contrast agents for $\mathrm{T}_{1}$ and $\mathrm{T}_{2}$ magnetic resonance imaging. Nanoscale. 2014;6(5):2953-2963.

40. Schultz-Sikma EA, Joshi HM, Ma Q, et al. Probing the chemical stability of mixed ferrites: implications for magnetic resonance contrast agent design. Chem Mater. 2011;23(10):2657-2664.
International Journal of Nanomedicine

\section{Publish your work in this journal}

The International Journal of Nanomedicine is an international, peerreviewed journal focusing on the application of nanotechnology in diagnostics, therapeutics, and drug delivery systems throughout the biomedical field. This journal is indexed on PubMed Central, MedLine, CAS, SciSearch ${ }^{\circledR}$, Current Contents ${ }^{\circledR} /$ Clinical Medicine,

\section{Dovepress}

Journal Citation Reports/Science Edition, EMBase, Scopus and the Elsevier Bibliographic databases. The manuscript management system is completely online and includes a very quick and fair peer-review system, which is all easy to use. Visit http://www.dovepress.com/ testimonials.php to read real quotes from published authors. 